\title{
edoc
}

Institutional Repository of the University of Basel

University Library

Schoenbeinstrasse 18-20

CH-4056 Basel, Switzerland

http://edoc.unibas.ch/

\section{Das Bedürfnis nach Sicherheit als Grundmotiv der Denkgeschichte}

\author{
Angehrn, Emil
}

Posted at edoc, University of Basel

Official URL: http://edoc.unibas.ch/dok/A6008471

Originally published as:

Angehrn, Emil. (2012) Das Bedürfnis nach Sicherheit als Grundmotiv der Denkgeschichte. In: Resilienz in der offenen Gesellschaft : Symposium des Centre for Security and Society. Baden-Baden, S. 71-83.

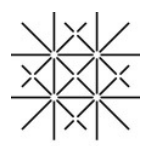




\section{Das Bedürfnis nach Sicherheit als Grundmotiv der Denkgeschichte}

\section{Emil Angehrn}

Das Bedürfnis nach Sicherheit ist ein basaler anthropologischer Sachverhalt. Wir begegnen ihm in unterschiedlichsten Bereichen des menschlichen Lebens: im Verhältnis des Menschen zu sich selbst, zu seinem Körper, zu anderen Menschen, zur umgebenden Natur, im Umgang mit der Technik, mit ökologischen und wirtschaftlichen Risiken, im Verhältnis zur Zukunft. Zur Nichtbeherrschbarkeit der Natur kommt die Nichtkontrollierbarkeit der vom Menschen geschaffenen Welt hinzu. Ebenso vielseitig wie die Gefährdungen sind die Sicherheitsdispositive - von Lawinenverbauungen über Gesundheitsregeln, Rechtssysteme und Polizeiapparate bis zu Beschwörungsritualen und technischen Apparaturen. Bemerkenswert ist im Blick auf die vielfältigen Formen des Umgangs mit Sicherheit zweierlei. Auffallend ist zum einen die historische Veränderung und typologische Vielfalt in der Artikulation des Sicherheitsbedürfnisses. Sie wirft nicht nur die Frage auf, wieweit wir hier überhaupt mit einem einheitlichen Phänomen und einer zusammenhängenden Fragestellung zu tun haben. Ebenso stellt sich die Frage, wieweit wir im historischen Wandel mit einer bestimmten Veränderung - Intensivierung, Ausweitung, Abschwächung der kulturellen Wahrnehmung des Sicherheitsproblems oder eher mit einer anthropologischen Konstante zu tun haben. Bezeichnend ist zum anderen die hohe Ambivalenz in der Beschreibung des Phänomens. Sicherheit wird ebenso selbstverständlich als individuelles Recht, gesellschaftlicher Wert oder technisches Ziel proklamiert wie sie - im Blick auf die psychische Verfassung wie die technischen Maßnahmen oder die sozialen Folgen - kritisch wahrgenommen wird. Auf der einen Seite erheben Sozialutopien und Menschenrechtserklärungen Sicherheit zum hohen Gut, auf der anderen Seite wird die primäre Sicherheitsorientierung als zwanghafte Fixierung oder existenzielle Verfehlung interpretiert, werden Grenzen und Selbstwidersprüche sozialer und technischer Sicherheitsstrategien aufgezeigt. Das zweifache Verhältnis von Varianz und Konstanz einerseits, von positiver und negativer Wertung andererseits scheint die Sicherheitsproblematik als ganze zu kennzeichnen.

Ich werde in den folgenden Überlegungen von diesem vielfältigen thematischen und historischen Befund einen Schritt zurücktreten und nach den allgemeinen Denkformen fragen, in denen uns das Motiv in der Denkgeschichte entgegentritt. Dabei ist von einem erstaunlichen Befund auszugehen. Auch wenn wir uns abstraktesten Fragen der Erkenntnistheorie und Ontologie zuwenden, zeigt sich eine bemerkenswerte Präsenz, ja, ein zentraler Stellenwert des Sicherheitsgedankens. Ich will dieser Präsenz in zwei Hauptschritten nachgehen. Zum einen geht es darum, das Motiv der Sicherheit im Kontext der allgemeinsten Leitfragen, über welche die beginnende Philosophie sich definiert, aufzuweisen. Bevor Sicherheit zum Gegenstand von Ethik und Politik wird, bestimmt sie die Fragen nach dem wahren Wissen und nach dem Sein. Zum anderen ist diese Verschränkung auf ihren anthropologischen Grund 
zurückzuführen und zu fragen, inwiefern das Streben nach Sicherheit zum Kern des menschlichen Seins gehört.

\section{Das Streben nach Sicherheit und die Suche nach Erkenntnis}

Die europäische Denkgeschichte scheint zuinnerst mit dem Streben nach Sicherheit verbunden. ${ }^{1}$ Die entstehende Philosophie ist in ihrem Kern durch die Suche nach Erkenntnis und Wahrheit definiert. In dieser Suche schließen die für die Folge maßgeblichen Konzepte von Platon und Aristoteles an eine Vorgeschichte an, die sich über die vorsokratischen Denker hinaus in das frühe Denken des Mythos hinein erstreckt. Aus diesen drei Stadien seien einige Leitmotive festgehalten, in denen die entstehende philosophische Reflexion sich in prägnanter Weise mit der Suche nach Sicherheit verschränkt. ${ }^{2}$

\subsection{Mythos: Die Überwindung des Chaos und das Standgewinnen in der Welt}

In seiner Schrift Arbeit am Mythos hat Hans Blumenberg das Anliegen des Mythos dahingehend charakterisiert, dass es darum geht, Stand zu gewinnen angesichts der Übermacht des Wirklichen - durch Benennung des Namenlosen, Unterscheidung des Diffusen, Gestaltung des Formlosen. Das undurchdringliche Ganze verliert seine Bedrohlichkeit, wenn wir in ihm Dinge identifizieren und auseinanderhalten, Strukturen und Regeln ausmachen, die es verlässlich und berechenbar, gegebenenfalls beherrschbar werden lassen. ${ }^{3}$ Idealtypisch begegnen wir solchen Figuren in den Weltentstehungsmythen, die den Übergang vom Chaos zum Kosmos, vom dunklen Urzustand zur geordneten Welt schildern. Hesiods Theogonie markiert diesen Übergang im ersten Satz der genealogischen Erzählung: „Wahrlich, zuallererst entstand das Chaos, später aber die breitbrüstige Erde, für alle Zeiten sicherer Sitz von allem. “" ${ }^{4}$ Das Chaos, das in zahllosen Varianten dieses weltweit verbreiteten Mythologems als Abgrund, undurchdringliches Dunkel und klaffende Leere, als unendlicher Raum, formloser Urstoff oder Urgewässer beschrieben wird, ist der Inbegriff dessen, was als bedrohlich und ängstigend erfahren wird. Die Welt entsteht, die Dinge ge-

1 Wie es sich mit der Denkform anderer Kulturen verhält, muss hier außer Betracht bleiben. Als Beispiel sei auf die chinesische Kultur verwiesen, in welcher François Jullien gerade mit Bezug auf die für unser Thema zentrale aristotelische Weichenstellung (siehe unten 1.3) eine alternative Denkform aufweist: Si parler va sans dire: Du logos et d'autres ressources, Paris: Seuil 2006.

2 Vgl. zum Folgenden ausführlicher: Emil Angehrn, Die Überwindung des Chaos. Zur Philosophie des Mythos, Frankfurt am Main: Suhrkamp 1996; Der Weg zur Metaphysik. Vorsokratik, Platon, Aristoteles, Weilerswist: Velbrück Wissenschaft 2000.

3 Hans Blumenberg, Arbeit am Mythos, Frankfurt am Main: Suhrkamp 1979.

4 Hesiod, Theogonie, V. $116 \mathrm{f}$. 
winnen Gestalt, indem sie aus dieser Zone der Auflösung herauskommen, sich vom Abgrund losreißen und sich im Widerstand gegen den Rückfall in das Nichtsein behaupten. Die für das griechische Denken charakteristische Aversion vor dem Grenzenlos-Unbestimmten ist eine Angst vor dem Nichtsein, die ganz auf das Bestimmte, Begrenzte, fest Umrissene setzt. Das Herausgehen aus dem Chaos ist das Gewinnen des sicheren Fundaments (des, für alle Zeiten sicheren Sitzes von allem'), die Eröffnung der Welt, in welcher alles seine feste Gestalt und seinen bestimmten Ort hat.

Diese in der Sukzession von Chaos und Erde inszenierte Urpolarität wird im Fortgang der Theogonie in unterschiedlichen Prozessen und Figuren durchgespielt. Ein erstes ist der Gegensatz zwischen den Nachkommenschaften der beiden Urpotenzen, den Mächten des Dunkels und der Zerstörung einerseits, den lichten Göttern andererseits, mit dem sich die mehrfach ausgetragenen Kämpfe zwischen alten und neuen Göttern, Mächten der Natur und des Geistes, verbinden, deren Ausgang schließlich die Herrschaft des Zeus über die Unterwelt, der stabilen Seins- und Rechtsordnung über die Kräfte der Auflösung bildet. Eine andere Figur ist die der Trennung, der Teilung, der Setzung von Grenzen. Vier der Schöpfungstage der Genesis sind mit Teilungen befasst (zwischen Licht und Finsternis, Tag und Nacht, Wasser und Erde etc.); eine der in allen Kulturen verbreitetsten Figuren ist die Trennung von Himmel und Erde, die den Ursprung des Lebens als Einbrechen des Lichts und Eröffnung des freien Raums versinnbildlicht. Die Angst vor dem Einsturz des Himmels ist die Urangst vor der Rückgängigmachung der Trennung, dem Rückfall in das Chaos, gegen welche die Theogonie die Schöpfung durch eine unüberwindliche Begrenzung der Unterwelt - die „,marmornen Pforten und die eherne, unerschütterlich feste Schwelle ${ }^{\text {c6 }}$ - befestigen will, die jede Vermischung zwischen den Welten und jede Auflösung von Bestimmtheit und Ordnung unterbinden soll. Seinswerdung heißt nicht nur Herauskommen des Seienden aus dem Nichtsein, Sichablösen der Gestalt aus dem Dunkel, sondern Halt und Festigkeit gewinnen, Widerstand leisten gegen den Rückfall und sich absichern gegen die Mächte der Destruktion und der Destabilisierung.

\subsection{Parmenides: Das reine Sein jenseits von Mischung und Wandel}

Es ist bemerkenswert, in welcher Prägnanz analoge Figuren bei den ersten Denkern der philosophischen Frühgeschichte wiederkehren. Herausragendes Beispiel ist das Lehrgedicht des Parmenides. Es steht ganz im Horizont eines höheren Wissens, das dem Dichter von der Göttin verkündet wird, welche ihm sowohl das „unerschütterliche Herz der wirklich überzeugenden Wahrheit” offenbart wie die „Meinungen der

5 Hesiod, Theogonie, V. $700 \mathrm{ff}$.

6 Hesiod, Theogonie, V. 811f. 
Sterblichen, denen keine wahre Verlässlichkeit innewohnt". In bezeichnender Weise tritt das wahre Wissen von Anfang an nicht nur mit dem Anspruch auf tiefste Einsicht, auf ein Vordringen ins Innerste des Wirklichen auf. Ebenso erhebt es den Anspruch auf letzte Sicherheit und Verbindlichkeit, die aber ihrerseits nicht in methodischen Regeln, sondern in der Festigkeit und inneren Stabilität des Wirklichen selbst ihren Grund finden. Parmenides formuliert als erster die Korrelation zwischen der Sicherheit - Verlässlichkeit, Überzeugungskraft - des wahren Wissens und der inneren Stabilität - Unerschütterlichkeit, Festigkeit - des Gewussten, die dann für die metaphysische Tradition mit Platon und Aristoteles verbindlich wird.

Konkret führt Parmenides dies in seiner Lehre vom Sein aus, die sich durch zweierlei auszeichnet: durch die strenge Trennung zwischen Sein und Nicht-Sein und durch die spezifischen Merkmale, anhand deren er das Sein kennzeichnet, wobei sich beides direkt in den Horizont des Sicherheitsgedankens rücken lässt. Das eine ist die strikte Trennung der Wege der Wahrheit und des Irrtums, welche die Göttin als Wege des Seins und des Nicht-Seins spezifiziert: Denken heißt Seiendes denken, Sprechen heißt sich auf etwas beziehen, was ist; denkend oder sprechend sind wir mit dem Wirklichen verbunden und finden in ihm allein, sofern es wirklich seiend ist, verlässlichen Halt. Was das Lehrgedicht in archaischen Formulierungen etwa im Diktum, dass Denken und Sein dasselbe sei ${ }^{8}-$ festhält, ist Ausdruck einer grundlegenden Intuition, die sich ebenso in der näheren Charakterisierung des Seienden als solchen niederschlägt, in welcher Parmenides den emphatischen Begriff eines reinen Seins, das von jeder Durchmischung mit Nichtseiendem und Scheinhaftem frei ist, zu erfassen sucht. Es ist die Idee eines bestimmten, mit sich identischen, in sich erfüllten, absolut gegenwärtig Seienden jenseits von Zeit und Bewegung, eines Seienden, das in keiner Weise innerlich brüchig oder unstabil, wandelbar und schwankend wäre. Solche Identität ist Grundlage von Verlässlichkeit: Sich sicher fühlen heißt, auf die feste Bestimmtheit wie auf die Konstanz der Menschen und Dinge zählen können.

Allerdings kann bereits dieser früheste Text der entstehenden Metaphysik auch als Sinnbild für die Zwiespältigkeit eines auf Sicherheit und Stabilität abzielenden Denkens gelesen werden. Profilierte Deutungen haben das Fragwürdige der einseitigen Festlegungen im parmenideischen Wirklichkeitsverständnis herausgestellt. Die rigorose Abtrennung zwischen Sein und Nicht-Sein erscheint dann als lebensweltlich begründete Angstbewältigung angesichts der Zweideutigkeiten und Instabilitäten des realen Lebens, das diese festen Grenzen zwischen Wahrheit und Schein, Licht und Dunkel nicht kennt; die Elimination solcher Ambivalenzen zugunsten eindeutiger Grenzziehungen erscheint im Zeichen der Abwehr. ${ }^{9}$ Generell kann die ge-

7 Parmenides, in: H. Diels/W. Kranz (Hg.), Die Fragmente der Vorsokratiker, 3 Bde, Berlin: 61951-1952, 28B1.29f.

8 Ebd., 28B3.

9 Klaus Heinrich, Parmenides und Jona. Vier Studien über das Verhältnis von Philosophie und Mythologie, Basel/Frankfurt am Main: Stroemfeld 1982; ders., tertium datur. Eine religions- 
waltsame Abdrängung des Wandels in das Reich des Uneigentlich-Unwahren als zutiefst befremdender Grundzug metaphysischen Denkens wahrgenommen werden. ${ }^{10}$ Der Charakter des Gewaltsam-Zwanghaften ist in Parmenides' eigener Beschreibung nicht zuletzt dort greifbar, wo es um das feste Mit-sich-Identischsein und -bleiben des Seins geht, von dem es heißt, dass es durch Dike, die Göttin des Rechts,

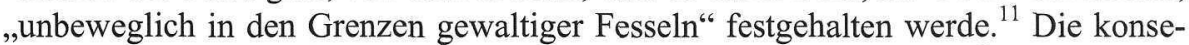
quente Entmischung von Sein und Nichtsein, die Ausmerzung der Übergänge und Zwischenbereiche soll den Phänomenen ihre Unheimlichkeit nehmen; die Eliminierung alles Fließenden und Zweideutigen soll die Zentrierung auf das Wahre und Feste ermöglichen und darin sicheres Wissen begründen - und läuft gleichzeitig Gefahr, solche Verlässlichkeit um den Preis der Verdrängung der realen Lebenswirklichkeit zu erkaufen.

\subsection{Platon und Aristoteles: Die Suche nach den ersten Prinzipien}

Unverkennbar definiert das Motiv, das den parmenideischen Thesen zugrunde liegt, eine Hauptstoßrichtung der entstehenden Metaphysik. Es bestimmt eine Hauptlinie im Denken von Platon und Aristoteles, die in ihren Grundlagentheorien, der platonischen Ideenlehre wie der aristotelischen Metaphysik, hervortritt und das darin anvisierte höchste Wissens in signifikanter Weise mit Aspekten der Identität und Bestimmtheit verbindet, wobei beidemal die Festigkeit in der Sache mit der Sicherheit im Erkennen einhergeht. Die Kontinuität und Spezifizierung dieses Motivs sei an wenigen Aspekten verdeutlicht.

Der ontologische Dualismus bildet den Kern und das Fundament der platonischen Ideenlehre. Die Differenz zwischen Ding und Idee ist als Transzendenz im epistemologischen wie im ontologischen Sinne gefasst: Die Ideen sind jenseits der sinnlichen Erfahrung, und sie sind das Andere gegenüber den einzelnen Dingen. Was in der Seinsweise beider Entitäten dem Gegensatz zugrunde liegt, wird analog zur Beschreibung bei Parmenides gefasst. Die basalste Kennzeichnung zielt auf den Gegensatz von Wandel und Konstanz: Das Jenseitige ist zunächst das Unveränderliche und Zeitlose. Die Ideen sind wie das parmenideische reine Sein ohne Entstehen und Vergehen, das Mit-sich-Identische und Sich-gleich-Bleibende; und wie bei Parme-

philosophische Einführung in die Logik (Dahlemer Vorlesungen 1), Basel/Frankfurt am Main: Stroemfeld 1981.

10 Eugen Fink, Zur ontologischen Frühgeschichte von Raum - Zeit - Bewegung, Den Haag:

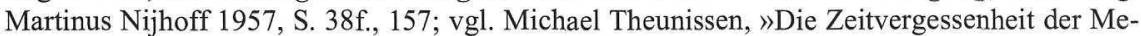
taphysik. Zum Streit um Paarmenides, Fr. 8.5-6a«, in: Negative Theologie der Zeit, Frankfurt am Main: Suhrkamp 1991, S. 89-130; Friedrich Nietzsche, Die Philosophie im tragischen Zeitalter der Griechen, in: Sämtliche Werke. Kritische Studienausgabe, München/Berlin: de Gruyter 1980, Bd. 1, S. 799-872.

11 Parmenides, Fragment B8.14f., 26, 30f.; vgl. dazu Hermann Schmitz, Der Ursprung des Gegenstandes. Von Parmenides bis Heraklit, Bonn: Bouvier 1988. 
nides ist Erkenntnis in dem Maße sicher und wahr, wie sie mit wahrhaft Seiendem, nicht mit Scheinhaftem, Brüchigem oder Fragmentarischem zu tun hat. Das wahrhaft Seiende ist das Eine, Identische und durch seine Wesensform Bestimmte, das sich vom Vielfältigen, Wandelbaren und Bestimmungslosen abhebt. Jede Uneindeutigkeit in seiner Bestimmtheit ist eine Minderung im Sein und zugleich eine Abschwächung der Erkennbarkeit. Vom Undeutlichen, Schwankenden, so die tragende Intuition, gibt es keine klare und verlässliche Erkenntnis. Die Erkennbarkeit gründet in der Seinsqualität des Gegenstandes, so dass es höchste Erkenntnis nur vom höchsten Seienden, von den Ideen, geben kann; unzweifelhaftes Wissen ist nur von dem möglich, was in sich selbst fest, unerschütterlich und klar bestimmt ist.

In besonders eindrucksvoller Weise bringt Aristoteles das Sicherheitsmotiv in der Letztbegründung der Wissenschaft zum Tragen. Im vierten Buch der Metaphysik kommt er auf jenes Prinzip zu sprechen, das nach ihm das grundlegendste Gesetz aller Wissenschaften ist und das als Prinzip des ausgeschlossenen Widerspruchs ausformuliert worden ist. Das Gesetz, dass man nicht dasselbe sowohl behaupten wie bestreiten kann, ist das allererste Prinzip, das die Konsistenz des Denkens und Argumentierens sichern soll. Aristoteles widmet dem Prinzip eine ausführliche Besprechung - was angesichts dessen erstaunen könnte, dass es um die Rechtfertigung eines Prinzips geht, von dem es zugleich heißt, dass es von allen das sicherste und am besten erkennbare sei, ja, dass es in Wahrheit von allen geteilt werde und dass alle Wissenschaftler in ihren Beweisen letztlich auf es zurückgreifen. Bemerkenswert ist die eindringliche Präsenz der Sicherheitsmetaphorik in der aristotelischen Explikation dieses nicht auf ein Früheres rückführbaren, ,voraussetzungslosen Anfangs'. Dass innerhalb weniger Sätze fünfmal die Umschreibung wiederkehrt, das erste Prinzip sei das sicherste von allen ${ }^{12}$, macht deutlich, dass das Interesse nicht einfach einem allgemeinsten Denkgesetz gilt, sondern ebenso sehr auf den festen Halt und unerschütterlichen Grund geht. Die Erkennbarkeit der Welt, statt über die interne Schlüssigkeit oder die Evidenz methodischer Regeln stabilisiert zu werden, wird gleichsam mit Bezug auf die ,reale' Begründung des in sich ruhenden Fundaments definiert. Das Erste ist nicht nur negativ als dasjenige gefasst, was nicht in einem anderen gründet, sondern positiv als dasjenige, was seinen Grund in sich selber hat und kraft dessen Stütze für anderes sein kann. Der feste Boden, der zum Gegenbild den Abgrund und den Sturz in die haltlose Leere hat, wird ex negativo noch in der Wortbedeutung von Sicherheit (asphaleia, Nicht-Stürzen) evoziert.

Nicht uninteressant ist in diesem Zusammenhang auch der Hinweis auf den Leitbegriff, mit welchem Aristoteles wie Platon den Ursprung der Philosophie aus dem Sichwundern beschreiben, wobei der Begriff nicht, wie zuweilen assoziiert, das Bewundern der kosmischen Ordnung, sondern das Irritiert- und Verwirrtsein angesichts eines Unverständlichen meint. Die Sonnenfinsternis oder die irrationale Zahl sind solche Anlässe, in denen ein etabliertes Weltverständnis und Weltvertrauen erschüt-

12 Aristoteles, Metaphysik 1005b9-22. 
tert wird und das Bedürfnis nach einem gründlicheren Erkennen erwacht. ${ }^{13}$ Wie sehr das von Aristoteles genannte Beispiel der irrationalen Zahlen mit Verstörung und Verunsicherung einhergeht, ist noch in den antiken Berichten greifbar, wonach derjenige aus dem Kreis der Pythagoreer, der die Entdeckung der Irrationalität an die Öffentlichkeit verraten habe, aus der Gemeinschaft der Schüler verstoßen worden oder sogar im Meer umgekommen sei. ${ }^{14}$ Nicht weniger aussagekräftig ist Platons Einführung des thaumazein als Ursprung der Philosophie, wenn er die Verwunderung über das Rätselhafte in die Nähe des Schwindels stellt ${ }^{15}$; Schwindligwerden (skotodineo) verbindet die Bedeutungskomponenten des Dunkels mit der des Wirbels oder Abgrunds - klassische Motive des Unheimlichen und der Angst. Angst im Dunkel wird durch das Licht der Erkenntnis vertrieben; sicheren Halt bieten die Festlegung fester Bezugspunkte und die Strukturierung anhand von Gesetzen. Je fundamentaler die Gründe sind, desto umfassender und desto sicherer ist die durch sie gewährte Transparenz und Stabilisierung.

Nur ergänzend seien andere Fluchtpunkte des metaphysischen Denkens genannt, in denen sich dessen Absehen auf Sicherheit kristallisiert. Ein prominentes Beispiel ist der Naturbegriff, der von alters her in klassischen Oppositionen begegnet, so in den Gegensätzen physis-nomos und physis-techne: Als Gegenbegriff zum Gesetz und zur Kunst kommt Natur einerseits als Geltungsgrund, anderseits als Entstehungsgrund in den Blick: Etwas ist hervorgebracht durch Natur oder durch menschliche Kunst, und es gilt von Natur oder aufgrund subjektiver Vereinbarung. In beiden Hinsichten fungiert physis in gewisser Weise als „Kontingenzabwehrbegriff $^{\star s 16}$ : Beidemal soll die Willkür subjektiver Setzung überwunden und diese an ein Ansichseiendes als Vorbild und Grund zurückgebunden werden. Die Natur als das aus sich Kommende und in sich Begründete ist ein Ort der Festigkeit und des Schutzes. Doch geht die Orientierung an Sicherheit weiter als die Ausrichtung auf das Ansichseiende und auf die Substanz. Sie kann auch die Gegenbewegung hin zum Subjekt, die Ermächtigung des Subjekts und seine Erhebung zum zentralen Referenzpunkt unseres Weltverständnisses bestimmen. Der moderne Mensch, der über die Entwicklung der Technik die Natur beherrscht ${ }^{17}$ und ihre Gefährdungen überwindet oder im politischen Handeln die Instabilitäten des Sozialen steuert ${ }^{18}$ und Herrschaft über die Geschichte erstrebt, wird mit der Utopie einer selbstverantworteten und eigenmächtig realisierten Sicherheit assoziiert. Martin Heidegger hat dies auf die Geschichte zurückprojiziert und Metaphysik im Ganzen durch das Sicher-

13 Aristoteles, Metaphysik 982b12-14, 983a13-17.

14 H. Diels/W. Kranz (Hg.), Die Fragmente der Vorsokratiker, a.a.O., 18.4.

15 Platon, Theaitetos $155 \mathrm{c}$.

16 Niklas Luhmann, Soziale Systeme. Grundriss einer allgemeinen Theorie, Frankfurt am Main: Suhrkamp 1984, S. 424.

17 Francis Bacon, Novum Organum (1620).

18 Thomas Hobbes, Leviathan (1651). 
heitsstreben subjektiver Herrschaft bestimmt. ${ }^{19}$ Ihre profilierte Ausprägung gewinnen solche Elemente außerhalb der hier verfolgten Fragestellung im Kontext der Politik und der Technik, von denen sie ihrerseits in die Grundlagenreflexion zurückspiegeln.

\section{Der existenzielle Kern: Der Umgang mit Angst und Kontingenz}

Mehrfach ist in den Umschreibungen des Sicherheitsgedankens dessen anthropologische Basis sichtbar geworden. Ein Blick auf den existenziellen Kern des Strebens nach Sicherheit kann dessen Fundamentalität im menschlichen Selbst- und Weltverhältnis verdeutlichen.

\subsection{Formen der Angst}

Das Streben nach Sicherheit erwächst aus der Angst. Thomas Hobbes, nach eigenem Zeugnis als Zwillingsbruder der Angst geboren - seine Mutter, durch die Nachricht von der Invasion der Armada erschreckt, „did bring forth Twins at once, both Me, and Fear ${ }^{r 20}$-, ist nicht nur Theoretiker des starken Staats und der Sicherheit, sondern auch der Angst: „So nagt die Furcht vor Tod, Armut oder einem anderen Unglück den ganzen Tag über am Herzen des Menschen, der aus Sorge über die Zukunft zu weit blickt, und er hat vor seiner Angst nur im Schlaf Ruhe. ${ }^{621}$ Angst ist der Affekt, auf den sich Politik am verlässlichsten abstützen kann, und zugleich das zu Überwindende; der Gewinn von Sicherheit ist Befreiung von Angst. Wovor aber fürchtet sich die Angst, wogegen bietet die Sicherheit Schutz? Ersichtlich handelt es sich hier nach beiden Seiten um ein äußerst vielgestaltiges, facettenreiches Phänomen. Wenn wir die Frage auf das Sichersein von Personen einschränken (im Gegensatz zur Sicherheit einer technischen Anlage, eines sozialen Systems), so können wir die Angst sowohl hinsichtlich ihrer gegenständlichen Korrelate wie des Status dieser Gegenstände differenzieren. Gegenstände der Angst können innere oder äußere, soziale, technische und natürliche Gegebenheiten sein; die Differenz im Status ist die Differenz zwischen realer Übermacht, aktueller bzw. mehr oder weniger konstanter Bedrohung, latenter Bedrohung und potenzieller Schädigung, Kontingenz und Unvorhersehbarkeit als solcher. Sicherheit kann erworben werden durch technische

19 Martin Heidegger, „Überwindung der Metaphysik“, in: Vorträge und Aufsätze, Pfullingen: Neske 1954, S. 67-96.

20 Thomas Hobbes, The Life of Mr. Thomas Hobbes of Malmesbury. Written by Himself in a Latine Poem, and now Translated into English (London 1680), S. 2; zit. nach: Thomas Hobbes, Leviathan, hrsg. und eingeleitet von Iring Fetscher, Darmstadt/Neuwied: Luchterhand 1966/Frankfurt am Main: Suhrkamp 1984, S. XI.

21 Thomas Hobbes, Leviathan, a.a.O., S. 83. 
Macht, durch Besitz, Rüstung und Planung, durch Versicherungen und institutionelle Regelungen; solchen Vorkehrungen einer funktional spezifizierten, pragmatisch oder technisch regulierten Absicherung steht die globale, nur begrenzt herstellbare soziale Sicherheit der 'Geborgenheit', aber auch die (Selbst-)Sicherheit durch Verwurzelung und Vertrauen gegenüber.

Bemerkenswert ist das Gefälle im Spektrum der Gestalten von Angst und Sicherheit. Erscheint zunächst die direkte Konfrontation mit äußerer Übermacht als das in unmittelbarster Weise Bedrohliche, so zeigt sich ebenso die mögliche Gefährdung, schließlich die innere Kontingenz als tieferer Grund der Angst. Die seit Kierkegaard geläufige Unterscheidung zwischen gegenstandsbezogener Furcht und gegenstandsloser Angst benennt einen Aspekt dieser Differenz; die Überführung der diffusen Angst in die bestimmte Furcht - die Eingrenzung und Identifizierung des Bedrohlichen, gegen das man sich zur Wehr setzen kann - ist eine Form der Angstbewältigung. Als innerster Kern zeigt sich in dieser Linie das Gewahrwerden der Nichtfestgelegtheit seiner selbst, der Haltlosigkeit aufgrund des Fehlens objektiver Stützen und eigener fester Bestimmtheit: Angstauslösend ist dann die Konfrontation mit der eigenen Freiheit (während umgekehrt das Absehen auf Sicherheit als Flucht vor der Freiheit erscheinen kann). Es gibt ein Leiden an äußerer und innerer Unbestimmtheit, eine Unfähigkeit zum Aushalten der Ambivalenzen, auf welche dann mit zwanghafter Fixierung, Eingrenzung, Selbstbindung reagiert wird. Das Bewusstwerden der realen Freiheitspotenziale, des Anwachsens der technischen und zivilisatorischen Möglichkeiten kann ein Gefühl der Unheimlichkeit - und einen entsprechenden Bedarf nach (ideologischer, politischer) Konsolidierung - erzeugen.

Angst als basale, im Subjekt selbst wurzelnde Befindlichkeit ist von der modernen Existenzphilosophie beschrieben worden. Heidegger nimmt den Gegensatz zur Furcht als Angelpunkt: Angst hat nicht nur mit Unbestimmtem, sondern mit der „wesenhaften Unmöglichkeit der Bestimmbarkeit“, der Erfahrung des Entgleitens, der Unheimlichkeit zu tun. ${ }^{22}$ Ähnlich hatte Kierkegaard auf die Unbestimmtheit der Freiheit für sich selber verwiesen und die Angst mit dem Gewahrwerden der Freiheit für sich selbst zusammengebracht. Seine Charakterisierung der Erfahrung des Schwindels wird von Sartre dahingehend spezifiziert, dass die Angst nicht dem möglichen Sturz in den Abgrund (als objektivem Ereignis), sondern der Möglichkeit des Sich-Hinunterstürzens, der Ungeschütztheit der Freiheit durch sich selber gilt. ${ }^{23}$ Die Nichtdeterminiertheit meiner Zukunft durch mein gegenwärtiges Ich macht meine Existenz ungesichert; nie kann ich sicher sein, dasjenige, was ich sein will, wirklich zu sein, dazwischen ist der Sprung der Freiheit, der jede Bestimmtheit zur Möglichkeit verflüchtigt. Es liegt nahe, dass auch psychologische Untersuchungen die untergründige Verbindung zwischen dem Angstaffekt und dem Sicherheitsbedürfnis herausstellen. Stellvertretend sei auf Michael Balint verwiesen, der die Angst

22 Martin Heidegger, „Was ist Metaphysik?“, in: Wegmarken, Frankfurt am Main: Klostermann 1967, S. 1-20 (9).

23 Jean-Paul Sartre, L'être et le néant, Paris: Gallimard 1943, S. 69. 
auf den Verlust der ursprünglichen Einheit des Subjekts mit seiner Umwelt zurückführt, mit welcher zugleich die ursprüngliche Sicherheit und Geborgenheit entfällt eine Erfahrung, die nicht rückgängig gemacht werden kann, sondern nur indirekt und partiell durch Wiederholung des ursprünglichen Traumas, im Verlassen der Sicherheitszone und im Eingehen von Trennungen und Risiken mit dem Ziel ihrer Wiedergewinnung bewältigt wird (exemplarisch im ,thrill', der ,Angstlust', auch in Extremsportarten und in Spielen aller Art, die das Aufgeben und Wiedererlangen von Sicherheit praktizieren). ${ }^{24}$ Aufschlussreich sind solche Beschreibungen nicht zuletzt hinsichtlich der Ambivalenz der affektiven Besetzung und existenziellen Erfahrung sowohl im Blick auf die Freiheit wie die Sicherheit, die beide erstrebt wie Gegenstand von Angst sein können. Die Sicherheit durch Wiedergewinnung der Einheit kann Halt gewähren oder in regressiven Selbstverlust münden.

\subsection{Machtsteigerung und Ausschluss der Zukunft}

Global gesehen, scheint es zwei entgegengesetzte Formen der Reaktion auf das Gewahrwerden von Kontingenz zu geben: Das Vermeiden und Überwinden von Kontingenzen einerseits, ihr Akzeptieren und Einbeziehen in die menschliche Existenz andererseits. Die typischen Formen des Sicherheitsstrebens bedienen sich der ersten Reaktionsweise, die wiederum in zweierlei Weise vorgehen kann, über die Steigerung eigener Macht und Handlungsmöglichkeiten oder über die Einschränkung der zu gewärtigenden Eventualitäten. Sichersein kann heißen, sich auf alles einstellen oder nur weniges zulassen, selber mächtig, idealiter allmächtig werden oder sein Gegenüber entmächtigen.

Die erste Grundform ist in Anthropologie, Ethik und Politik seit Platon mannigfach beschrieben worden, zumeist mit kritischem Unterton als Zeichen der Selbstverfehlung und des Verfalls. Besitzstreben und Machtstreben sind ihre klassischen Ausprägungen. Die in ihnen angelegte Tendenz zur schlechten Unendlichkeit führt dazu, dass die Akkumulierung der Möglichkeiten letztlich dysfunktional wird und die Realisierung des ursprünglich erstrebten Ziels unterläuft. Die zwanghafte Absicherung gegen alle erdenklichen Bedrohungen kann den Wert des zu Schützenden gegenüber der Effizienz des Sicherheitssystems selbst zurücktreten lassen. Die reflexiv-indirekte Struktur gehört zur Logik der Sicherheit: Es geht dann nicht mehr um den direkten Schutz von etwas, sondern darum, die Zuverlässigkeit des Schutzes zu gewährleisten. Solche Sicherheitslogik enthält eine zweifache Unzulänglichkeit. Sie ist zum einen prinzipiell begrenzt und letztlich aporetisch: Auch über zwei- und mehrstufige Sicherungen lässt sich keine absolute Sicherheit herstellen; Frieden

24 Michael Balint, Angstlust und Regression. Beitrag zur psychologischen Typenlehre, Hamburg: Rowohlt 1972. Ähnlich beschreibt E. Erikson das Urvertrauen als das relative Gleichgewicht mit dem lebenslänglichen Kontrapunkt eines Ur-Misstrauens: Erik H.L. Erikson, Identität und Lebenszyklus, Frankfurt am Main: Suhrkamp 1976, S. 69. 
muss man letztlich halten, nicht sichern. Zum andern ist die Indirektheit und Reflexivität offen für die Verkehrung zum Selbstzweck, Ansatzpunkt der Dysfunktionalität und schlechten Unendlichkeit: Der Sicherheitsbedarf als nie zu stillendes Bedürfnis ist von Wirtschaft und Industrie längst als neue Ressource entdeckt worden, die sich über die Erweiterung des Wissens von Risiken und die veränderte Definition von Grenzwerten und Toleranzschwellen fast unerschöpflich steigern lässt.

Die andere, gegenläufige Variante der Kontingenzvermeidung ist die Festschreibung des zu Erwartenden, die Fixierung im Raum der Möglichkeiten. Wenn Unsicherheit in Kontingenz gründet, wird Sicherheit durch den Ausschluss des Andersseinkönnens realisiert. Der Umgang mit dem Formlos-Unbestimmten als Gegenstand der Angst wird durch Festlegung auf Bestimmtheit und Starrheit des Verhaltens überwunden. Im Ausschluss des Andersseinkönnens hat Sicherheit eine unmittelbar temporale Komponente. Das Interesse an der Stabilität einer Institution ist Teil des Interesses an der Sicherheit, die sie gewährleisten soll. Seit je bildet es einen Kernpunkt der Politik, wie die Ordnung zu erhalten, vor Verfall zu bewahren sei - wobei die sicherheitsorientierte Antwort dahin geht, die Erhaltung über den Ausschluss des Wandels zu garantieren. Fast alle Sozialutopien behandeln Stabilität als vorrangiges Ziel und tendieren bezeichnenderweise zur Starrheit. Die Erfahrung des Unvorhergesehenen ist Prototyp der Kontingenzerfahrung; deren rudimentärste Überwindung ist der Ausschluss der Neuheit. So ist Sicherheit nicht nur temporal bestimmt, sondern wesentlich auf die Suspendierung eines Wesenszugs der Zeit, der Offenheit der Zukunft angelegt. Je weniger die Verfügung über Zukunft durch fiktive Allmacht zu gewährleisten ist, desto näher liegt der Versuch, sich des Kommenden durch Negation seines Zukunftscharakters zu bemächtigen. Unzulänglich und aporetisch sind beide Vorgehen gleichermaßen.

\subsection{Anerkennung und Integration der Kontingenz}

So scheint der einzige Weg zur Sicherheit darin zu bestehen, Kontingenz nicht auszuschalten, sondern sie zu integrieren, sich auf sie einzustellen. Dies geschieht teils in gegenständlichen Verhaltensweisen, teils in Einstellungsänderungen, die eine andere Grundhaltung zum Nichtkontrollierbaren, aber auch erhöhte Lern- und Reaktionspotenziale zum flexiblen Umgang mit Eventualitäten beinhalten. Dass der flexible Umgang mit Risiken höhere Sicherheit gewährleisten kann als die rigide Risikoprävention, liegt in der für menschliches Leben konstitutiven Ungewissheit, die ausschließt, dass Sicherheit ein für allemal erworben werden kann. Zum Umgang mit Risiko gehört das Akzeptieren eigener Endlichkeit und Fehlbarkeit: Angst und Ängstlichkeit - auch die Furcht davor, sich im Nachhinein etwas vorwerfen zu müssen - können sich selbst als Gefahr erweisen.

Die verlangte Einstellungsänderung ist eine im Umgang mit sich und der Welt: Es geht nicht um das Tolerieren von Restrisiken, sondern das Akzeptieren grundsätzlicher Kontingenz - nicht als Grundlage passiver Indifferenz, sondern von Eigenver- 
antwortung, Klugheit, Hoffnung. Allerdings lässt auch die so umrissene Haltung tiefgreifende Alternativen in der Beschreibung und Wertung offen. Soll der sachgerechte Umgang mit Kontingenz als angemessenes Sicherheitsverhalten oder als ein Verhalten jenseits des Sicherheitsstrebens, als Zulassen oder Überwinden der Angst charakterisiert werden? Für N. Luhmann wie für postmoderne Denker hat das heute geforderte Umgehen mit Kontingenz deren Negativität (als Relikt einer alteuropäischen Wertordnung) hinter sich gelassen ${ }^{25}$; wenn andererseits $H$. Lübbe Religion und Historie als Kulturen der Kontingenzbewältigung beschreibt, so klingen darin die lebensweltlichen Schwierigkeiten des Umgangs mit Unsicherheit an, die solche Kulturleistungen erforderlich machen ${ }^{26}$ Das Beschwören des Aushaltenkönnens von Kontingenz und Bodenlosigkeit nimmt zum Teil einen Gestus des Heroischen an, der seinerseits problematisierbar ist. Dagegen kann gerade das Zulassen von Angst als realitätsgerechte und existenziell authentische Einstellung gefordert werden, kann das Insistieren auf Angstfreiheit als Maske und Angstabwehr durchschaut werden. Hier geht das Sicherheitsproblem in den Kampf der Definitionen über: vom Kampf um die Festschreibung von Grenzwerten bis hin zum Streit darüber, ob der Maßstab des humanen Umgangs mit Kontingenz in einer wahren Sicherheit oder einem Zustand jenseits der Sicherheit liege. Deutlich aber ist, dass es sich dabei nicht um einen bloßen Streit um Worte handelt.

\section{Fundamentalität und Ambivalenz der Suche nach Sicherheit}

Die Frage nach der Sicherheit, nach dem richtigen Umgang mit Angst und Kontingenz erweist sich als eine Frage, die unser Verhältnis zur Welt, aber auch unser Verständnis des Menschen, die Frage, wie wir uns selbst verstehen und wie wir unser Leben führen wollen, zuinnerst betrifft. Thre Ausformulierung zeigt im Ganzen nicht nur die Ambivalenz in der Wahrnehmung und kulturellen Beschreibung des Sicherheitsphänomens. Sie weist ebenso auf eine bestimmte Aporetik in dessen konsequenter Entfaltung, und dies sowohl in der erkenntnistheoretisch-ontologischen wie der anthropologisch-praktischen Version.

In beiden Bereichen folgt die Suche nach Sicherheit zunächst einem Grundmuster, dessen konsequente Ausformulierung seine Problematik aufzeigt. Dieses Grundmuster besteht darin, das Problem, auf welches das Sicherheitsbedürfnis antwortet, auszuschalten - es aktiv zu bewältigen oder es zu leugnen, zu verdrängen, zu unterlaufen. Die Verunsicherung durch das Unbestimmte, der Schrecken vor der Leere, die Angst vor der Kontingenz werden in dieser Grundhaltung dadurch überwunden, dass gegen sie ein fester Grund, ein eindeutig Bestimmtes und mit sich Identisches, ein Dauerhaftes, Notwendiges und Geordnetes gesucht wird, das dem Subjekt Halt

25 Richard Rorty, Kontingenz, Ironie, Solidarität, Frankfurt am Main: Suhrkamp 1992.

26 Hermann Lübbe, Religion nach der Aufklärung, München: Fink 32004. 
bietet und Orientierung ermöglicht. Halt verschaffen soll eines, das in sich selbst stabil und fest begründet ist.

Gegen dieses Grundmuster, das einen Hauptstrang der Denkgeschichte im Theoretischen wie im Praktischen bestimmt, melden andere Strömungen Bedenken an, und dies aus zwei verschiedenen Gründen. Zum Teil insistieren sie auf der Nichtrealisierbarkeit dieser Strategie, der Nichterreichbarkeit der intendierten Sicherheit, die entweder das menschliche Vermögen überfordert oder sich in ihrer konsequenten Durchsetzung selbst behindert und widerspricht. Das Lebendig-Wandelbare durch Rekurs auf starre Festlegungen zu schützen ist Beispiel einer solchen sich selbst aufhebenden Teleologie. Zum Teil aber betonen sie nicht die Unerreichbarkeit, sondern die Nichtwünschbarkeit des Telos, indem sie die Sicherheit als leitenden Wert problematisieren und dagegen das angemessene Umgehenkönnen mit Unsicherheit und Kontingenz, das Vermögen, sich im Offenen und Haltlosen zu bewegen, als die dem Menschen gemäße Lebensform und als adäquate Reaktion auf die Herausforderung durch Unbestimmtheit und Unsicherheit sehen. Gegebenenfalls vertreten sie die Meinung, dass gerade so wahre Sicherheit erreichbar sei: Die Flechte, nicht der lineare Faden, die bunte Vielfalt, nicht die monotone Systematik geben dem Leben Halt. Ähnlich formulieren andere Positionen den Vorbehalt im Theoretischen gegenüber einem identifizierend-klassifizierenden Denken, gegen welches sie für alternative Formen der Wirklichkeitserschließung im Medium des Vielfältig-Offenen plädieren.

Bemerkenswert scheint in dieser Gesamtkonstellation zweierlei. Das eine ist die Fundamentalität des Sicherheitsgedankens, der nicht erst in der ethisch-praktischen und politischen Dimension virulent wird, sondern schon im theoretischen Weltbezug sich als grundlegend erweist und in ontologischen Optionen zum Ausdruck kommt. Das andere ist die Strukturanalogie in der Aporetik und in dem ihr korrespondierenden Doppelweg des Umgehens mit Unsicherheit im erkenntnistheoretischen wie im existenziell-praktischen Wirklichkeitsverhältnis. Sie spricht dafür, dass es sich hier um einen in der Sache liegenden Grundzug des Problems der Sicherheit und des Umgangs mit ihr handelt. Die Divergenz und interne Gegenläufigkeit der beiden Wege der Überwindung und der Zulassung von Kontingenz durchzieht die Ideengeschichte, in welcher keine der beiden Seiten die andere zu widerlegen, ihr Spannungsverhältnis aufzulösen vermochte. Es bleibt zu sehen, wieweit sich Aspekte dieser Konstellation in der konkreteren Ausführung des Sicherheitsgedankens im Rahmen der Rechts- und Sozialphilosophie widerspiegeln. 
